\title{
Numerical Simulation of Deformation-Induced Segregation in Continuous Casting of Steel
}

\author{
T. KAJITANI, J.-M. DREZET, and M. RAPPAZ
}

\begin{abstract}
The deformation-induced macrosegregation in continuous casting of steel has been simulated using a finite-volume scheme. For that purpose, a two-dimensional heat-flow computation was first performed in a Eulerian reference frame attached to the mold, assuming a unique solidification path, i.e., a unique relationship between temperature and enthalpy. This gave the stationary enthalpy field in the longitudinal section of the slab. On the other hand, bulging of the slab between two rolls was calculated in the same section, assuming plane-strain deformation and using the ABAQUS code. The Lagrangian reference frame was attached to the slab, and the rolls were moved at the surface until a stationary, bulging deformation profile was reached. The bulging of the surface was then used as an input condition for the calculation of the velocity and pressure fields in the interdendritic liquid. Using a fairly simple hypothesis for the deformation of the solid skeleton, the mass conservation and Darcy equations were solved in a Eulerian reference frame. This calculation was performed in an iterative loop, within which the solute conservation equation was also solved. At convergence and using the enthalpy field, this calculation allowed to obtain the temperature, the volume fraction of solid, and the average concentration fields, in addition to the fluid velocity and pressure. It is shown that the positive centerline segregation of carbon in the slab is well reproduced with this model. The effects of shrinkage and soft reduction were also investigated.
\end{abstract}

\section{INTRODUCTION}

MACROSEGREGRATION, i.e., an inhomogeneity of solute concentration at the scale of the whole product, is a common defect encountered in most solidification processes. It is always due to a relative velocity of the liquid with respect to the solid phase, and, as such, it can be induced by thermosolutal convection, ${ }^{[1,2]}$ forced convection ${ }^{[3]}$ solidification shrinkage, ${ }^{[4,5,6]}$ transport and sedimentation of grains, ${ }^{[7]}$ or deformation of the solid skeleton. ${ }^{[8,9]}$ In continuous casting of steel, bulging of the cast slab between two successive rolls has been shown to play a major role in the centerline segregation, which occurs at a fairly late stage of solidification. ${ }^{[8,9]}$ This phenomenon, which is schematically illustrated in Figure 1, can be explained as follows.

Assuming that the solid and liquid have the same specific mass (i.e., no solidification shrinkage), let us consider the stationary situation between two successive rolls of a continuous casting machine. The casting direction points toward the right, and the gravity vector direction is arbitrary. The ferrostatic pressure associated with the deep liquid sump at this position is such that the solid crust that has formed has a tendency to creep between the rolls. The resulting bulging of the slab (enhanced in Figure 1 for the purpose of illustration) makes solid material move out and then in of a rectangular domain outlined by the inner roll position. The velocity at any point within the solid crust is simply the sum of the

T. KAJITANI, formerly Research Fellow, Ecole Polytechnique Fédérale de Lausanne, is Senior Researcher with the Kimitsu R\&D Laboratory, Nippon Steel Corporation, Kimitsu, Kimitsu-city Chiba, Japan. J.-M. DREZET, Senior Researcher, is with Calcom SA, Lausanne, Switzerland, and Ecole Polytechnique Fédérale de Lausanne. M. RAPPAZ, Professor, is with the Laboratoire de Métallurgie Physique, Département des Matériaux, Ecole Polytechnique Fédérale de Lausanne, CH-1015 Lausanne, Switzerland

Manuscript submitted February 2, 2000. horizontal casting speed, $\mathbf{v}_{c}=v_{c} \mathbf{e}_{x}$, and the vertical bulging speed, $\mathbf{v}_{b}=v_{c} \tan \alpha \mathbf{e}_{y}$, where $\alpha$ is the angle that the bulged surface makes locally with the horizontal (Figure 1(a2)).

For the sake of clarity, let us now assume that the temperature field is such that the position of the liquidus at the beginning of this small section $\left(T_{L, i n}\right)$ is at the center of the slab. If there was no heat extraction at the surface of the slab, the isotherms would follow the bulging profile (Figure 1(a1)). Accordingly, the position of the liquidus at the exit of the domain $\left(T_{L, \text { out }}\right)$ would also be at the center of the slab. The velocity field being always tangent to the liquidus surface (Figure 1(a2)), * the mass balance integrated over

\footnotetext{
*From a mass-balance point of view, the velocity of the liquid within this pocket could be set equal to zero everywhere, except at the boundary where it is tangent. This will ensure that mass is conserved for any closed contour of this domain. However, there will then be a discontinuity in the velocity field at the boundary. Considering the mass and momentum conservation equations, the situation shown in Figure 1(a) would lead to two recirculation vortices within the liquid pocket.
}

this surface (white area in Figure 1(a1)) is equal to zero. This system, therefore, does not need to exchange mass with the solute-rich interdendritic liquid and no macrosegregation is induced (i.e., a closed system). When heat is extracted at the surface of the slab (Figure 1(b1)), solidification proceeds during bulging, and the point $T_{L, o u t}$ has now moved some distance ahead of the exit rolls. The velocity field imposed by the casting and bulging speed is no longer tangent to the liquidus surface (Figure 1(b2)). Since $\mathbf{v}_{s} \cdot \mathbf{n}>0$, where $\mathbf{n}$ is the outward pointing normal to the liquidus surface, there is a deficit of mass over the liquidus surface. In other words, some solute-rich interdendritic liquid has to enter this area in order to compensate for this loss: this liquid is expulsed from the compression of the mushy zone in the region located between $T_{L, \text { out }}$ and $T_{S, \text { out }}$ (Figure 1(b1), i.e., in the region where the dendrites growing from both sides entangle). This effect, which can be viewed as the compression of a 


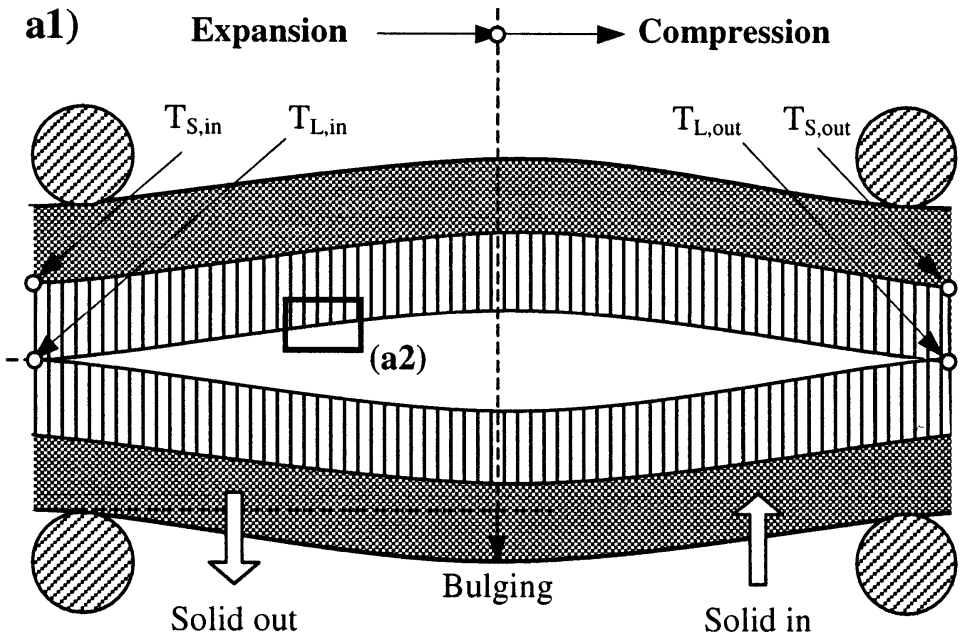

b1)

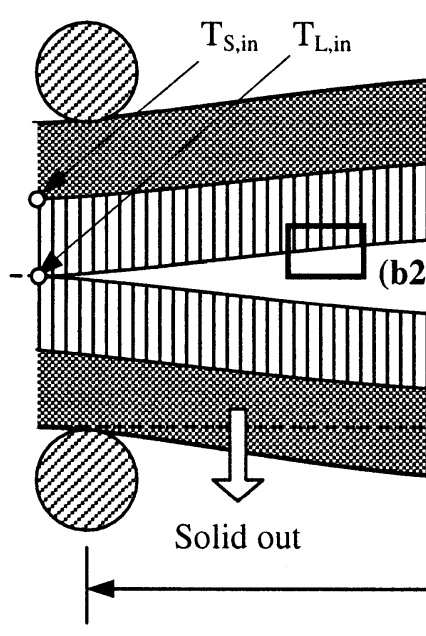

Casting direction

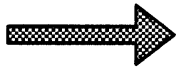

Solid in

Liquid
ШाI] Mushy
$\mathrm{T}_{\mathrm{L}, \mathrm{out}}$

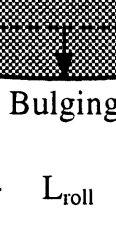

$\mathrm{L}_{\text {roll }}$
ulging

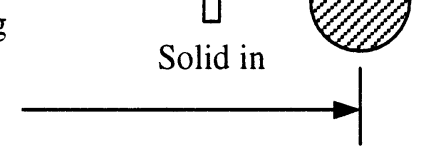

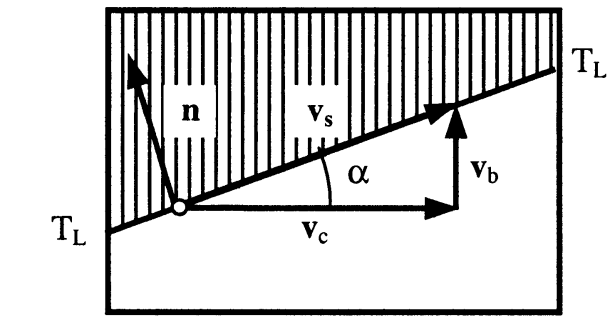

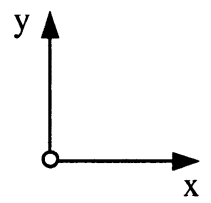

b2)

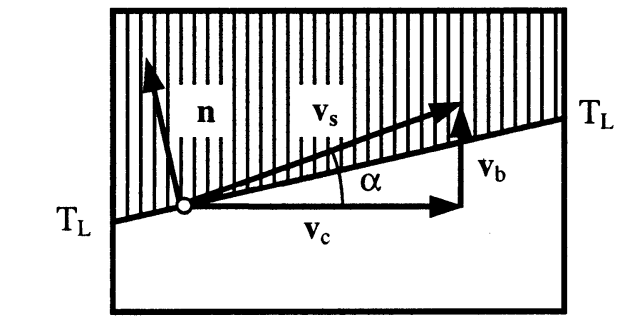

Fig. 1-Schematics of bulging between two rolls during continuous casting of steel, when there is still some liquid near the centerline, for $(a)$ adiabatic boundary condition and $(b)$ heat extracted at the surface of the slab.

sponge,${ }^{[9]}$ will be shown to occur repeatedly between successive rolls as the fraction of solid increases (i.e., even when the extradendritic liquid of Figure 1 has disappeared). It is the main reason for the centerline segregation observed in continuous casting of steel, ${ }^{[8]}$ together with the solidification shrinkage ${ }^{[8]}$ and thermal strains of the coherent solid of the mushy zone. ${ }^{[9]}$

Several experimental techniques have been devised in order to reduce the positive centerline segregation in cast steel. In-mold electromagnetic stirring ${ }^{[10]}$ promotes the fragmentation of dendrites by local remelting and, thus, enhances the formation of fine equiaxed grains, predominantly at the center of the slab. These grains tend to sediment, and segregation is dispersed between them. Another means is the socalled "soft reduction" applied during the final stage of solidification. ${ }^{[11]}$ In this technique, the steel slab is progressively compressed by the rolls in order to compensate for the solidification shrinkage.

Numerical simulation of macrosegregation in continuous casting of steel slabs is made difficult by the interplay of all these phenomena: bulging, shrinkage, soft reduction, and grain sedimentation. Yet, it would be a very valuable help to operate a continuous casting machine. To our knowledge, a comprehensive model in this area does not yet exist. Miyazawa and Schwerdtfeger ${ }^{[8]}$ pioneered this field and calculated the solute distribution by solving, in a fully Eulerian approach, the combined mass- and solute-conservation and Darcy equations. Although the predicted solute distribution seemed to be in fairly good agreement with the experiment, the liquid velocity obtained near the axis of symmetry of the slab did not comply, apparently, with the boundary condition. This might be due to the streamline formulation used by these authors for solving the average conservation equations. More recently, using an approach very close to that of Miyazawa and Schwerdtfeger, $\mathrm{Li}^{[12]}$ could only reproduce the influence of shrinkage on macrosegregation. The predicted contribution of bulging was very weak and not very well localized at the center of the slab. Lesoult and Sella ${ }^{[9]}$ used 


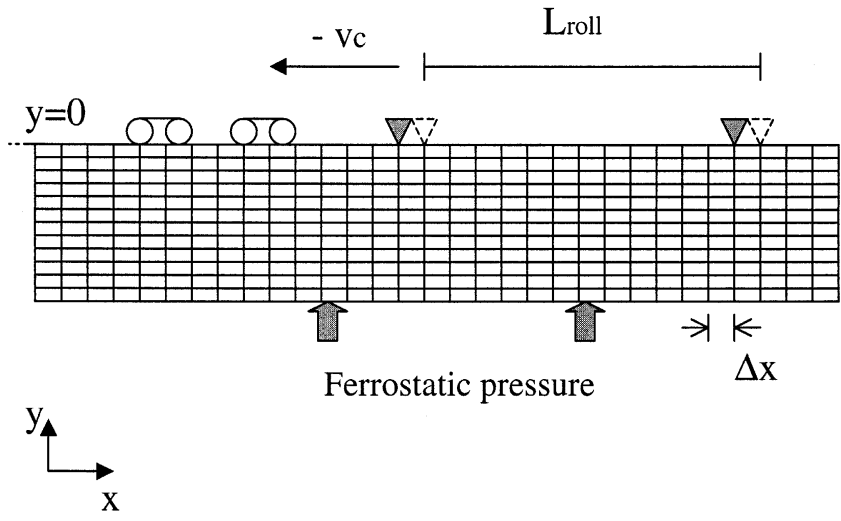

Fig. 2-Finite-element enmeshment and boundary conditions used for the stress-strain analysis.

a mixed Eulerian-Lagrangian approach, in which the conservation equations were formulated in a reference frame attached to the solid skeleton and deforming with it. This approach provides an interesting means of extending the macrosegregation criterion established by Flemings and $\mathrm{Nereo}^{[4]}$ to the case of solid-skeleton deformation. The importance of the differential thermal contraction between the surface and heart of the slab on macrosegregation could be evidenced by these authors, but they neglected bulging and did not fully couple the conservation equations. This formulation is also more difficult to implement in a numerical simulation and, thus, limits the applications.

In the present study, macrosegregation induced by bulging, shrinkage, and soft reduction is calculated in a fully Eulerian approach (grain sedimentation and thermal contraction are neglected). However, instead of using a streamline formulation, the fluid flow induced by the various contributions is calculated by solving the pressure field. As will be shown, the solution satisfies the boundary condition imposed at the center of the slab. The stationary bulging profile that forms between two rolls is first calculated in a Lagrangian representation using the ABAQUS ${ }^{[13]}$ finite-element method (FEM) code and a fairly simple approach, as compared with previous models. ${ }^{[14,15]}$ This bulging profile is then used as an input for the segregation calculation. Besides a careful calculation of the fluid-flow velocity field, this model offers the advantages that several rolls can be considered and that the influences of both shrinkage and soft reduction can be quantitatively assessed. It will be shown in particular that each bulging-compression sequence between two successive rolls contributes to the centerline segregation increase. Section II briefly describes the mechanical model used to calculate bulging, whereas the macrosegregation model is detailed in Section III. Simulation results are presented and discussed in Section IV.

\section{BULGING MODEL}

In a first step, bulging of the solid crust between two successive rolls is calculated using the ABAQUS ${ }^{[13]}$ FEM code and a standard creep model. The calculation is performed by moving two rolls over a portion of the ingot surface which is much longer than the distance separating them, until a stationary bulging shape is obtained. The calculation domain with the enmeshment is shown in Figure 2: it is a rectangle with a thickness given by that of the solid crust only (the resistance to creep of the mushy zone is neglected). The free surface of the slab (top) is at atmospheric pressure, whereas a ferrostatic pressure is imposed on the bottom surface corresponding to the solidus. The action of the rolls is simulated by imposing the position of two nodes of the regular mesh moving in the opposite direction at a velocity of $v_{c}$. This is done during a time increment of $\Delta t$ $=\Delta x / v_{c}$, where $\Delta x$ is the mesh size in the $x$-direction. As the casting direction is toward the right (Figure 1), these Dirichlet boundary conditions are moved to the next mesh points located in the opposite direction after that time (Figure 2 ). This procedure is repeated until a stationary bulging shape is obtained between the two nodes. In order to avoid bulging on the two parts located outside the segment defined by these two points, a Dirichlet condition $y=0$ is also fixed for those regions.*

*The cylindrical rolls not only impose the $y$-position of the slab at the contact point, they also define the tangent of the surface (i.e., $d y / d x=0$ at the contact points). In a numerical code such as ABAQUS, this is usually achieved by also modeling the rolls and by assuming no penetration between the rolls and the slab. The maximum deformation slope near the contact points that can be achieved in the slab is then dictated by the finite size of the mesh used to enmesh the rolls. The resulting bulging being only of the order of $1 \mathrm{~mm}$, it was verified in a simulation that included the roll shape, that the no-penetration condition could be applied just at the contact points.

Since creep is the dominant deformation mechanism at elevated temperatures, the strain rate $(\dot{\boldsymbol{\varepsilon}})$ is written in terms of an elastic and viscoplastic contribution:

$$
\dot{\boldsymbol{\varepsilon}}=\dot{\boldsymbol{\varepsilon}}_{e l}+\dot{\boldsymbol{\varepsilon}}_{v p}
$$

Unlike Lesouldt and Sella, ${ }^{[9]}$ the thermal strain has been neglected, but the temperature dependence of the mechanical properties has been accounted for. The elastic contribution is related to the stress tensor $(\boldsymbol{\sigma})$ by Hooke's law:

$$
\dot{\boldsymbol{\varepsilon}}_{e l}=\mathbf{D}_{e l}^{-1} \dot{\boldsymbol{\sigma}}
$$

where $\mathbf{D}_{e l}$ is the elastic tensor defined by the Young's and Poisson's moduli. Neglecting primary creep, the viscoplastic strain rate can be written as:

$$
\dot{\boldsymbol{\varepsilon}}_{v p}=\frac{3 \dot{\varepsilon}_{v p}^{e q}}{2 \sigma^{e q}} \mathbf{S}
$$

where $\sigma^{e q}$ is the Von Mises' equivalent stress, $\mathbf{s}$ is the deviatoric stress tensor, and $\dot{\varepsilon}_{v p}^{e q}$ is the equivalent viscoplastic strain rate, defined by the following creep law: ${ }^{[16]}$

$$
\dot{\varepsilon}_{v p}^{e q}=A \exp \left(\frac{-Q}{\mathrm{R} T}\right)\left(\sin h\left(a_{\sigma} \sigma^{e q}\right)\right)^{n}
$$

The activation energy $(Q)$ and the other parameters of this viscoplastic law are given in Table I, together with the material properties used in the present simulation. ${ }^{[17]}$ Considering the very deep liquid sump associated with continuous casting of steel, the heat flow giving the temperature field in the crust was assumed to occur only in the direction perpendicular to the casting direction. The nonstationary temperature field $(T(y, t))$ was then converted to $T(x, y)$ according to the translation $x=v_{c} t$. The same calculation also provided the enthalpy field $(h(x, y))$ used in the macrosegregation model. 
Table I. Materials Properties and Casting Conditions Used in the Calculations

\begin{tabular}{|c|c|c|}
\hline & & Units \\
\hline Casting speed, $v_{c}$ & 1 & $\mathrm{~m} \mathrm{~min}^{-1}$ \\
\hline Thickness of steel slab & 200 & $\mathrm{~mm}$ \\
\hline Distance between rolls & 400 & $\mathrm{~mm}$ \\
\hline \multicolumn{3}{|l|}{ Heat-transfer coefficient at the surface } \\
\hline from 0 to $1 \mathrm{~m}$ below the free surface & 1000 & $\mathrm{~W} \mathrm{~m}{ }^{-2}{ }^{\circ} \mathrm{C}^{-1}$ \\
\hline from 1 to $3 \mathrm{~m}$ & 500 & $\mathrm{~W} \mathrm{~m}{ }^{-2}{ }^{\circ} \mathrm{C}^{-1}$ \\
\hline beyond $3 \mathrm{~m}$ & 300 & $\mathrm{~W} \mathrm{~m}{ }^{-2}{ }^{\circ} \mathrm{C}^{-1}$ \\
\hline external temperature & 30 & ${ }^{\circ} \mathrm{C}$ \\
\hline \multicolumn{3}{|l|}{ Mechanical properties } \\
\hline \multicolumn{3}{|l|}{ Young's modulus: $E$} \\
\hline$E(T)=4 \times 10^{4}-20 \times T$ & $\left(\mathrm{~T}\right.$ in $\left.{ }^{\circ} \mathrm{C}\right)$ & $\mathrm{MPa}$ \\
\hline Poisson's ratio: & 0.3 & - \\
\hline \multicolumn{3}{|l|}{ Creep law: $\dot{\varepsilon}_{v p}^{e q}=A \exp (-Q / \mathrm{R} T)\left(\sinh \left[a_{\sigma} \sigma^{e q}\right]\right)^{n}(T$ in $\mathrm{K})$} \\
\hline$A=1.802 \times 10^{6}+1.742 \times 10^{8} w_{s}-6.503 \times 10^{7} w_{s}^{2}$ & & $\mathrm{~s}^{-1}$ \\
\hline$Q=44809$ & & $\mathrm{~J}$ mole ${ }^{-1}$ \\
\hline$a_{\sigma}=1.068+1.702 \times 10^{-4} T-2.808 \times 10^{-7} T^{2}$ & & $\mathrm{MPa}^{-1}$ \\
\hline$n=0.2+3.966 \times 10^{-4} T$ & & - \\
\hline \multicolumn{3}{|l|}{ Thermophysical properties } \\
\hline Solute content (carbon), $w_{0}$ & 0.55 & wt pct \\
\hline Liquidus temperature, $T_{L}$ & 1470.7 & ${ }^{\circ} \mathrm{C}$ \\
\hline Solidus temperature, $T_{s}$ & 1345.7 & ${ }^{\circ} \mathrm{C}$ \\
\hline Melting point of pure iron, $T_{m}$ & 1536.0 & ${ }^{\circ} \mathrm{C}$ \\
\hline Slope of liquidus line, $m$ & -118.75 & ${ }^{\circ} \mathrm{C}$ wt pct ${ }^{-1}$ \\
\hline Partition coefficient, $k$ & 0.35 & - \\
\hline Specific mass of solid, $\rho_{s}$ & 7400.0 & $\mathrm{~kg} \mathrm{~m}^{-3}$ \\
\hline Specific mass of liquid, $\rho_{l}$ & 7000.0 & $\mathrm{~kg} \mathrm{~m}^{-3}$ \\
\hline Specific heat, $c_{p}$ & 686.0 & $\mathrm{~J} \mathrm{~kg}^{-1}{ }^{\circ} \mathrm{C}^{-1}$ \\
\hline Volume latent heat of fusion, $L$ & 2046. $\times 10^{6}$ & $\mathrm{~J} \mathrm{~m}^{-3}$ \\
\hline Viscosity of liquid, $\mu$ & 0.005 & $\mathrm{~Pa} \mathrm{~s}$ \\
\hline Permeability in the $x$-direction, $K_{x}$ & $2.19 \times 10^{-14} t_{f}^{1.7} g_{l}^{3.34}$ & $\mathrm{~m}^{2}$ \\
\hline Permeability in the $y$-direction, $K_{y}$ & $3.13 \times 10^{-13} t_{f}^{0.96} g_{l}^{2}$ & $\mathrm{~m}^{2}$ \\
\hline
\end{tabular}

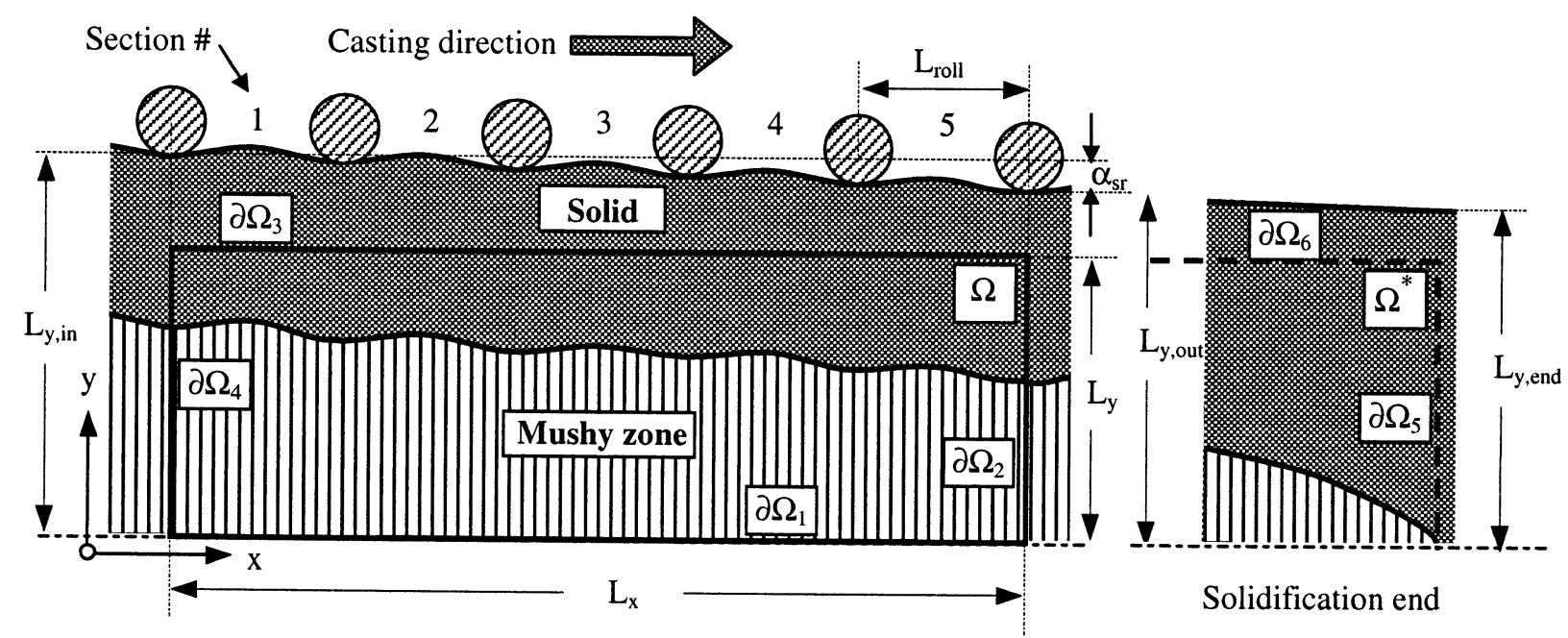

Fig. 3-Schematics of the domain $(\Omega)$ used to calculate deformation-induced segregation. With soft reduction $\left(\alpha_{s r}<0\right)$, an overall mass balance must be performed over the entire solidification domain $(\Omega \cup \Omega *)$.

\section{MACROSEGREGATION MODEL}

\section{A. Mass Conservation and Darcy Equations}

The macrosegregation model is two-dimensional and corresponds to the midsection of the slab perpendicular to the rolls (Figure 3). Assuming stationary conditions, the domain $(\Omega)$ within which conservation equations are written is rectangular and fixed. In the $x$-direction, it starts at some roll position and extends over a length of $L_{x}=N L_{\text {roll }}$, where $N$ is the number of intervals between rolls $(N=5$ in Figure 3 ). The dimension of the domain in the $y$-direction is $L_{y}$ and starts at the centerline. Unlike the schematic description shown in Figure 1, the domain only contains the mushy zone and a portion of the solid crust (i.e., the calculation is started once a certain fraction of solid is reached at the centerline position and no extradendritic liquid is left). The 
representation is fully Eulerian, i.e., the solid moves in and out of the domain $\Omega$ as the slab expands or is compressed (Figure 1). The fully solid crust is assumed to move with a velocity field $\left(\mathbf{v}_{s}(x)\right)$, given in the fixed reference frame by

$$
\begin{aligned}
\mathbf{v}_{s}(x) & =\mathbf{v}_{c}+\mathbf{v}_{y}(x)=v_{c} \mathbf{e}_{x}+v_{c} \tan \alpha(x) \mathbf{e}_{y} \\
& =v_{c}\left(\mathbf{e}_{x}+\tan \alpha(x) \mathbf{e}_{y}\right)
\end{aligned}
$$

where $\mathbf{v}_{y}(x)$ is the velocity of the crust in the transverse direction, given by the local angle $\alpha(x)$ between the bulged surface and the horizontal (a function only of $x$, Figures 1 and 3). If soft reduction is applied to the slab (Figure 3), this angle is given by

$$
\alpha(x)=\alpha_{b}(x)+\alpha_{s r}
$$

where $\alpha_{b}(x)$ is the angle calculated with ABAQUS from the stationary bulging profile without soft reduction (Figure 1), whereas the angle $\alpha_{s r}$, associated with soft reduction, is simply given by (Figure 3):

$$
\alpha_{s r} \approx \tan \alpha_{s r}=\frac{L_{y, \text { out }}-L_{y, \text { in }}}{L_{x}}\left(\alpha_{s r}<0 \text { in Figure } 3\right)
$$

where $L_{y, \text { in }}$ and $L_{y, \text { out }}$ are the half thicknesses of the slab at the entry $(x=0)$ and exit $\left(x=L_{x}\right)$ of the calculation domain, respectively.

The derivation of the equations describing fluid flow and macrosegregation within $\Omega$ follows quite closely that of Miyazawa and Schwerdtfeger. ${ }^{[8]}$ The stationary conservation equation of mass can be written as

$$
\operatorname{div}\left(\rho_{s} g_{s} \mathbf{v}_{s}+\rho_{l} g_{l} \mathbf{v}_{l}\right)=0
$$

or

$\operatorname{div}\left(\bar{\rho} \mathbf{v}_{s}+\rho_{l} g_{l} \mathbf{v}_{l, r}\right)=\operatorname{div}\left(\bar{\rho} \mathbf{v}_{s}+\rho_{l} g_{l}\left(\mathbf{v}_{l}-\mathbf{v}_{s}\right)\right)=0$

where $\rho$ is the specific mass, $g(x, y)$ is the volume phase fraction, and $\mathbf{v}(x, y)$ is the velocity field. The subscripts " $s$ " and "l" stand for the solid and liquid phases, respectively. The specific masses of the two phases are assumed to be constant, but not necessarily equal, and $\bar{\rho}=\left(\rho_{l} g_{l}+\rho_{s} g_{s}\right)$ is the average specific mass. The relationship between the relative velocity of the liquid phase $\left(\mathbf{v}_{l, r}=\left(\mathbf{v}_{l}-\mathbf{v}_{s}\right)\right)$ and the pressure $(P(x, y))$ in the mushy zone is described by Darcy's equation: ${ }^{[18]}$

$$
g_{l}\left(\mathbf{v}_{l}-\mathbf{v}_{s}\right)=-\frac{K}{\mu} \operatorname{grad} P
$$

where $K\left(g_{s}(x, y)\right)$ is the permeability of the mush, and $\mu$ is the dynamic viscosity of the liquid. Instead of using a standard Carman-Kozeny law, the permeability of columnar dendrites was taken from an empirical relationship that differentiates the longitudinal and transverse components. ${ }^{[12]}$ This relationship is given in Table I as a function of the volume fraction of liquid $\left(g_{l}=1-g_{s}\right)$ and of the local solidification time $\left(t_{f}\right)$. Instead of using a streamline formulation, ${ }^{[8,12]}$ the velocity in the liquid is eliminated by combining Eqs. [8] and [9]:

$$
\begin{gathered}
\operatorname{div}\left(\bar{\rho} \mathbf{v}_{s}\right)-\operatorname{div}\left(\rho_{l} \frac{K}{\mu} \operatorname{grad} P\right)=\mathbf{v}_{s} \cdot \operatorname{grad} \bar{\rho} \\
+\bar{\rho} \operatorname{div} \mathbf{v}_{s}-\operatorname{div}\left(\rho_{l} \frac{K}{\mu} \operatorname{grad} P\right)=0
\end{gathered}
$$

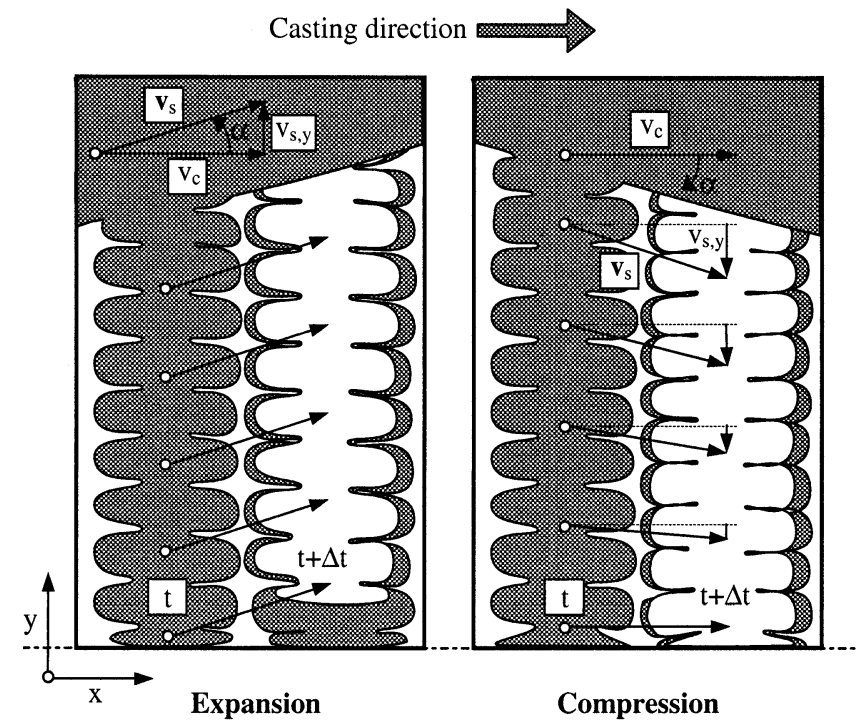

Fig. 4-Schematics of the movement of a columnar dendrite between time ( $t$ ) (in gray) and time $(t+\Delta t)$ (in white), during the expansion and compression stages of bulging.

It is interesting to note that the liquid flow, given by the pressure term, can be induced either by gradients of the average specific mass (i.e., shrinkage) or by a non-divergence-free field of the solid movement (i.e., $\operatorname{div} \mathbf{v}_{s} \neq 0$, deformation of the solid skeleton). This elliptical equation for the pressure field, $P(x, y)$, can be solved if appropriate boundary conditions are given and the velocity of the solid phase is known. Equations [5] through [7] are assumed to be valid everywhere in the fully solid region (the gray area in Figure 3). The main problem is now to estimate the solid velocity in the mushy zone (the hatched region). Two distinct situations are considered (Figure 4):

(1) If there is local expansion at the position $x$ (i.e., $\alpha=$ $\alpha_{b}+\alpha_{s r}>0$ ), the solid dendrites are assumed to move with the same velocity as the solid crust, i.e., $\mathbf{v}_{s}(x)$ is uniform along a direction perpendicular to the casting direction and given by Eqs. [5] through [7]. In Figure 4 , a Lagrangian representation of the dendrite displacement is given: during the time step $\Delta t$, one columnar dendrite in gray moves by a quantity $\mathbf{v}_{s} \Delta t$ to a position shown in white. During the same time interval, it solidifies and becomes a little bit thicker (the gray area surrounding the white dendrite).

(2) If there is local compression at the position $x$ (i.e., $\alpha<$ $0)$, the dendrites are compressed non-uniformly. Since the vertical component of the velocity of the solid dendrites at the centerline position $(y=0)$ must be zero for symmetry reasons, $v_{s}(x, y)$ in the mushy zone is assumed to be given by a linear interpolation:

$$
\mathbf{v}_{s}(x, y)=\mathbf{v}_{c}+\mathbf{v}_{y}(x) \frac{y}{y_{s}(x)}=v_{c}\left(\mathbf{e}_{x}+\tan \alpha(x) \frac{y}{y_{s}(x)} \mathbf{e}_{y}\right)
$$

where $y_{s}(x)$ is the position of the solidus front at the position $x$. Accordingly, the portion of the dendrites located near the centerline has a horizontal velocity equal to the casting speed, whereas that located near the 
solidus moves with the crust. This situation is again illustrated in Figure 4 in a Lagrangian representation.

\section{B. Boundary Conditions}

The boundary conditions necessary to solve Eq. [10] need to be specified. At the centerline $\partial \Omega_{l}$ (Figure 3 ), it is assumed that there is no mass flow across the boundary. When $\mathbf{v}_{s}=$ 0 (the compression zone, Figure 4), this also implies that $\mathbf{v}_{l}=0$. This is equivalent to setting

$$
\frac{\partial P}{\partial n}=0 \quad \text { at } \partial \Omega_{1} \text { when } \alpha<0
$$

In the expansion zone (Figure 4), the velocity of the solid being not equal to zero at the centerline, the boundary condition becomes

$$
\frac{\partial P}{\partial n}=-\frac{\mu}{K} \frac{\bar{\rho}}{\rho_{l}} v_{s, y} \quad \text { at } \partial \Omega_{1} \text { when } \alpha>0
$$

When formulating this boundary condition with a finitevolume method, the contribution of the interdendritic liquid flow levels off that of the solid (i.e., no average mass transfer through the centerline). Therefore, at the centerline, the boundary condition $v_{s, y} \neq 0$ and Eq. [12b] can as well be replaced by $v_{s, y}=0$ and Eq. [12a].

The boundary condition on $\partial \Omega_{3}$ (Figure 3 ) is also set to

$$
\frac{\partial P}{\partial n}=0 \quad \text { at } \partial \Omega_{3}
$$

which is equivalent to setting $v_{l}=v_{s}$ (Eq. [9]). In the fully solid crust, Eq. [10] is solved using a penalty method: the permeability is set to an arbitrary, constant, small value. Since $\mathbf{v}_{s}$ is a divergence-free field in the solid crust, ${ }^{*}$ this

*The horizontal component of the solid velocity being uniform, only the vertical component has to be considered to prove that div $\mathbf{v}_{s}=0$. Since $v_{s, y}=v_{c} \tan \alpha(x)$, it can be shown that

$$
\begin{aligned}
\int_{\Omega} \operatorname{div} \mathbf{v}_{s} d \Omega & =\int_{\partial \Omega} v_{c} \tan \alpha(x) n_{y} d s \\
& =\int_{\partial \Omega} v_{c} \tan \alpha(x)\left(1 / \sqrt{1+y^{\prime 2}}\right)\left(\sqrt{1+y^{\prime 2}} d x\right) \\
& =\int_{\partial \Omega} v_{c} \tan \alpha(x) d x=0
\end{aligned}
$$

on any arbitrary, closed domain. Therefore, $\mathbf{v}_{s}$ is a divergence-free field.

implies that grad $P$ is also a divergence-free field when $K$ (and $\mu$ ) are constant (Eq. [10]). Accordingly, the velocity of the liquid is penalized to that of the solid in the whole solid crust.

The boundary conditions on the vertical boundaries $\partial \Omega_{2}$ and $\partial \Omega_{4}$ can be deduced from the overall mass balance integrated over the solidification domain. Since no mass is crossing the centerline $\partial \Omega_{1}$, the integration of Eq. [8] over $\Omega$ gives

$$
\begin{gathered}
\int_{\partial \Omega_{2}}\left(\rho_{s} g_{s} v_{s, x}+\rho_{l} g_{l} v_{l, x}\right) d y+\int_{\partial \Omega_{3}} \rho_{s} v_{s, y} d x \\
-\int_{\partial \Omega_{4}}\left(\rho_{s} g_{s} v_{s, x}+\rho_{l} g_{l} v_{l, x}\right) d y=0
\end{gathered}
$$

(The $x$-axis is pointing inward to the boundary $\partial \Omega_{4}$, which explains the negative sign in Eq. [14]). The net mass crossing the boundary $\partial \Omega_{3}$ is zero if there is no soft reduction. However, if soft reduction is applied, the second term of Eq. [14] can be estimated from

$$
\begin{aligned}
\int_{\partial \Omega_{3}} \rho_{s} v_{s, y} d x & =\rho_{s} v_{c} \tan \alpha_{s r} L_{x} \\
& =\rho_{s} v_{c}\left(L_{y, \text { out }}-L_{y, \text { in }}\right)\left(<0 \text { if } \alpha_{s r}<0\right)
\end{aligned}
$$

Please note that an assumption regarding the solid velocity has been made: the rolls turning at the same speed, the horizontal component has been assumed to be constant and equal to $v_{c}$, despite soft reduction. On the other hand, when the mass balance is integrated until the end of solidification (Figure 3, domain $\Omega \cup \Omega *$ ), one has

$$
\begin{aligned}
& \int_{\partial \Omega_{5}} \rho_{s} v_{c} d y+\int_{\partial \Omega_{3} \cup \partial \Omega_{6}} \rho_{s} v_{s, y} d x \\
& -\int_{\partial \Omega_{4}}\left(\rho_{s} g_{s} v_{s, x}+\rho_{l} g_{l} v_{l, x}\right) d y=0
\end{aligned}
$$

The first integral of Eq. [16] is simply equal to $\rho_{s} v_{c} L_{y}$, whereas the second one is given by $\rho_{s} v_{c}\left(L_{y, \text { end }}-L_{y, \text { in }}\right.$ ) (Figure 3). Subtracting Eq. [16] from [14], the mass balance over $\partial \Omega_{2}$ can be obtained as

$\int_{\partial \Omega_{2}}\left(\rho_{s} g_{s} v_{s, x}+\rho_{l} g_{l} v_{l, x}\right) d y=\rho_{s} v_{c}\left(L_{y, \text { end }}-L_{y, \text { out }}+L_{y}\right)$

and from that, the mass balance on $\partial \Omega_{4}$ :

$\int_{\partial \Omega_{4}}\left(\rho_{s} g_{s} v_{s, x}+\rho_{l} g_{l} v_{l, x}\right) d y=\rho_{s} v_{c}\left(L_{y, \text { end }}-L_{y, \text { in }}+L_{y}\right)$

In order to obtain a condition on the pressure, the relative velocity of the fluid $\left(\mathbf{v}_{l}-\mathbf{v}_{s}\right)$ first has to be introduced in Eqs. [17] and [18]. Since the horizontal component of the solid velocity is assumed to be equal to $v_{c}$ everywhere on $\partial \Omega_{2}, *$ one has

*The position of this boundary is located at a roll position (Figure 3 ).

$$
\begin{aligned}
\int_{\partial \Omega_{2}} \rho_{l} g_{l}\left(v_{l, x}-v_{s, x}\right) d y & =\rho_{s} v_{c}\left(L_{y, \text { end }}-L_{y, \text { out }}+L_{y}\right) \\
& -v_{c} \int_{\partial \Omega_{2}} \bar{\rho} d y
\end{aligned}
$$

Using Eq. [9] and assuming that the integral Eq. [19] can be applied locally at each point of the boundary $\partial \Omega_{2}$, Eq. [19] then gives

$\frac{-K}{\mu} \frac{\partial P}{\partial n}=v_{c}\left(\frac{L_{y, \text { end }}-L_{y, \text { out }}+L_{y}}{L_{y}} \frac{\rho_{s}}{\rho_{l}}-\frac{\bar{\rho}}{\rho_{l}}\right)$ at $\partial \Omega_{2}$

Similarly, Eq. [18] gives the local boundary condition:

$$
\frac{K}{\mu} \frac{\partial P}{\partial n}=v_{c}\left(\frac{L_{y, \text { end }}-L_{y, \text { in }}+L_{y}}{L_{y}} \frac{\rho_{s}}{\rho_{l}}-\frac{\bar{\rho}}{\rho_{l}}\right) \text { at } \partial \Omega_{4}
$$

Please note that when soft reduction is not applied (i.e., when $\alpha_{s r}=0$ or $L_{y, \text { in }}=L_{y, \text { out }}=L_{y, \text { end }}$ ), the two boundary Eq. [20] and [21] become 


$$
\begin{gathered}
\frac{-K}{\mu} \frac{\partial P}{\partial n}=v_{c}\left(\frac{\rho_{s}}{\rho_{l}}-\frac{\bar{\rho}}{\rho_{l}}\right) \text { at } \partial \Omega_{2} \\
\frac{K}{\mu} \frac{\partial P}{\partial n}=v_{c}\left(\frac{\rho_{s}}{\rho_{l}}-\frac{\bar{\rho}}{\rho_{l}}\right) \text { at } \partial \Omega_{4}
\end{gathered}
$$

If shrinkage is further neglected, then these two equations resume to Eq. [13]. In such a case, it is interesting to note that all the boundary conditions imposed on $\partial \Omega$ are of a homogeneous Neumann-type and are equivalent to stating that $\mathbf{v}_{l}=\mathbf{v}_{s}$. In other words, the domain $\Omega$ behaves as a closed system, as already explained for the particular case of a single pair of rolls in Figure 1. Consequently, when shrinkage is neglected and no soft reduction is applied, each section of the continuous caster contributes to macrosegregation independently of the others.

\section{Solute Conservation Equation and Coupling}

Knowing $v_{s}(x, y)$ and $v_{l}(x, y)$, the stationary conservation equation for the solute content of a binary alloy is given by

$$
\operatorname{div}\left(\mathbf{v}_{s} \rho_{s} g_{s} w_{s}+\mathbf{v}_{l} \rho_{l} g_{l} w_{l}\right)=0
$$

where $w_{s}(x, y)$ and $w_{l}(x, y)$ are the concentrations of solute in the solid and liquid phases per unit mass, respectively. Please note that diffusion at the macroscopic scale of the casting has been neglected. Assuming equilibrium, i.e., $w_{s}=k w_{l}$, where $k$ is the partition coefficient, and introducing the relative velocity of the fluid, one has:

$\operatorname{div}\left(\mathbf{v}_{s} w_{l}\left(\rho_{s} g_{s} k+\rho_{l} g_{l}\right)\right)+\operatorname{div}\left(\left(\mathbf{v}_{l}-\mathbf{v}_{s}\right) \rho_{l} g_{l} w_{l}\right)=0$

Since this equation is purely advective, the boundary conditions need to be specified only where the relative fluid velocity is entering the domain. This means that

$$
w_{l}(x, y)=w_{L}(T(x, y))=\frac{T(x, y)-T_{m}}{m} \text { at } \partial \Omega_{4}
$$

where $w_{L}(T)$ is the equation of the liquidus, $T_{m}$ is the melting point of pure iron, and $m$ is the slope of the liquidus. It is to be noted that this relationship is applied everywhere in the mushy zone, in order to relate the local temperature and solute concentration in the liquid. The problem has to be further completed by the relationship

$$
h(x, y)=\rho c_{p} T(x, y)+L\left(1-g_{s}(x, y)\right)
$$

where the enthalpy field, $h(x, y)$, is computed before starting the segregation calculation using appropriate thermal-boundary conditions (Table I). The terms $\rho c_{p}$ and $L$ are the volumetric specific heat and latent heat of fusion, respectively.

In an iterative procedure, the macrosegregation calculation is performed as follows

(1) The local concentration in the liquid $\left(w_{l}(x, y)\right)$, the temperature $(T(x, y))$, and the volume fraction of solid $\left(g_{s}(x, y)\right)$ are first initiated in the domain $\Omega$ according to a local lever rule and assuming that the average solute concentration is uniform and equal to the nominal concentration.

(2) The permeability $\left(K\left(g_{s}\right)\right)$ is computed according to the relationship given in Table I.

(3) Taking the bulging profile calculated in the previous section for all the segments of $\Omega$ and the assumed softreduction angle, the local pressure in the liquid $(P(x, y))$ is calculated according to Eq. [10] with the appropriate boundary conditions.

(4) The relative velocity of the fluid $\left(\mathbf{v}_{l}-\mathbf{v}_{s}\right)$ is computed from the pressure field using Eq. [9].

(5) The concentration in the liquid is calculated with the help of Eq. [25].

(6) The temperature field is updated with these new concentrations according to the liquidus equation.

(7) The volume fraction of solid is updated according to Eq. [27], keeping the enthalpy field unchanged. Using these new values, the iteration is continued at point (2) until convergence is reached.

The finite-volume method was used to solve Eqs. [10] and [25], and an upwind scheme was implemented in this last equation. The material properties and casting conditions used in the calculation are given in Table I. They correspond to a pseudobinary $\mathrm{Fe}-\mathrm{C}$ system with a nominal concentration of 0.55 wt pct.

\section{RESULTS AND DISCUSSION}

The macrosegregation results presented in this section were obtained for a domain that included six rolls (i.e., five sections of the continuous caster, Figure 3). Unless specifically mentioned in the text, the material properties as well as the solidification conditions used in the computations are listed in Table I. Prior to the macrosegregation calculation, a thermal calculation was performed in a one-dimensional configuration in order to obtain the enthalpy field $(h(y, t))$. This field was then converted into a stationary field: $h(x, y)=h\left(v_{c} t, y\right)$. The heat-transfer coefficient applied at the surface of the slab is also given in Table I as a function of the distance below the top free surface of the melt. The macrosegregation calculation was not performed for the entire mushy zone, mainly because of the computation time and convergence difficulty at very low and very high volume fractions of solid. The initial volume fraction of solid at the boundary $\partial \Omega_{4}\left(g_{s, 0}\right)$ was of the order of 0.2 to 0.6 at the centerline position (i.e., at $x=y=0$ ).

The typical velocity fields calculated under various conditions are presented in Figure 5 for the middle section (i.e., section 3 in Figure 3) of the domain. The casting speed, which is also the horizontal component of the solid velocity, is equal to $16.7 \mathrm{~mm} \mathrm{~s}^{-1}$ in all cases (toward the right-hand side). At the top of the figure, the displacement of the solid surface calculated with ABAQUS is also shown with a magnification in the $y$-direction (refer to the scale in Figure 5): the maximum bulging displacement is about $1 \mathrm{~mm}$. In Figure 5(a), shrinkage has been neglected (i.e., $\rho_{s}=\rho_{l}$ ), and no soft reduction has been applied (i.e., $\alpha_{s r}=0$ ). In this case, the relative velocity of the interdendritic fluid, $\mathbf{v}_{l}-\mathbf{v}_{s}$, is mainly in the opposite direction of the casting speed and presents a maximum near the middle of the centerline of the slab $\left(1.46 \mathrm{~mm} \mathrm{~s}^{-1}\right)$. As already mentioned in Section III-B, each section of the caster is almost independent of its neighbors in the sense that there is almost no mass exchange. Indeed, at each roll position, it can be verified that the horizontal component of the relative velocity of the fluid is almost zero. The velocity of the fluid also decreases as one moves away from the centerline, i.e., when the fraction of 


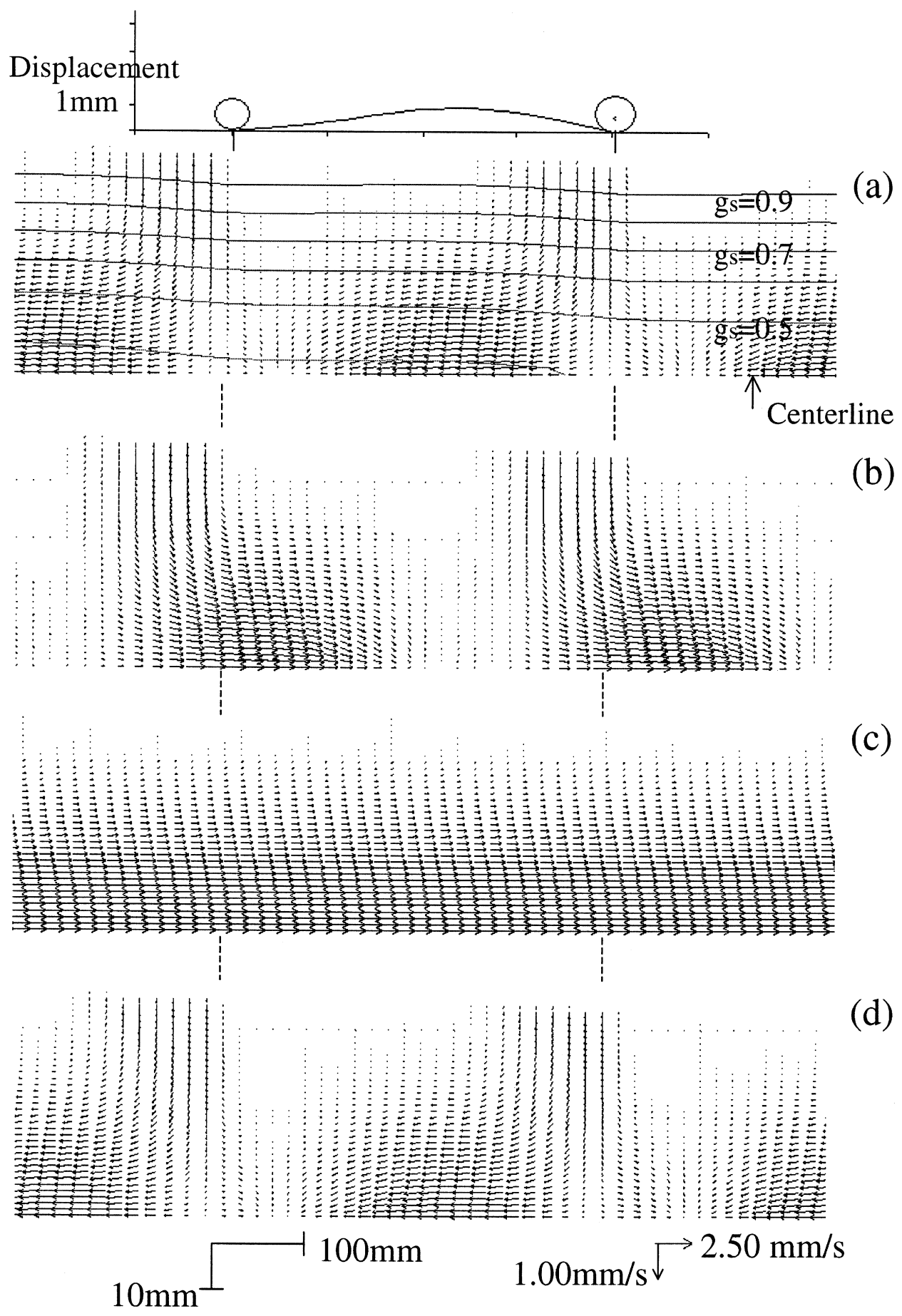

Fig. 5-Relative liquid velocity field $\left(\mathbf{v}_{l}-\mathbf{v}_{s}\right)$ in the mushy zone calculated with the macrosegregation model for various conditions: (a) maximum bulging of $1 \mathrm{~mm}$, no shrinkage $\left(\rho_{s}=\rho_{l}\right)$ and no soft reduction $\left(\alpha_{s r}=0\right) ;(b)$ maximum bulging of $1 \mathrm{~mm}$, with shrinkage $\left(\rho_{s} \neq \rho_{l}\right)$ and no soft reduction $\left(\alpha_{s r}=\right.$ $0) ;(c)$ no bulging, with shrinkage $\left(\rho_{s} \neq \rho_{l}\right)$ and no soft reduction $\left(\alpha_{s r}=0\right) ;(d)$ maximum bulging of $1 \mathrm{~mm}$, with shrinkage $\left(\rho_{\mathrm{s}} \neq \rho_{l}\right)$ and soft reduction $\left(\alpha_{s r}=-1.5 \cdot 10^{-4} \mathrm{rad}\right)$. The initial volume fraction of solid, $g_{s, 0}$, at the centerline position $(0,0)$ is $0.37(121 \times 45$ nodes $)$.

solid increases, the permeability decreases. Please note also that, unlike the results of Miyazawa and Schwerdtfeger, ${ }^{[8]}$ the present calculations satisfy the condition of no mass flux through the centerline (i.e., $v_{y}(x, y=0)=0$ ). As mentioned in the introduction (Figure 1), the interdendritic flow is initiated in the compression zone in order to feed the upper part of the section of the caster, which is in expansion. However, this occurs only when solidification proceeds at the same time, i.e., when the isoenthalpy lines are not exactly parallel to the surface of the slab. When macrosegregation is weak, the enthalpy is a unique function of the volume fraction of solid; consequently, isovolume fractions (shown as solid lines) have been drawn in Figure 5(a) instead of isoenthalpy ones.

Introducing now the difference between the specific masses of the solid and liquid phases, it can be seen that the flow pattern is considerably changed (Figure 5(b)). The compression introduced by the rolls still induces a negative 
component of the vertical velocity of the fluid (i.e., $v_{l, y}<$ 0 ), but the horizontal component is now in the casting direction (i.e., $v_{l, x}-v_{s, x}>0$ ) and is at a maximum at the roll position. This is because the liquid has to feed the mushy zone located downstream in the continuous caster in order to compensate for shrinkage. This can be easily understood by computing the relative fluid flow in the presence of shrinkage but without bulging (Figure 5(c)): in order to compensate for the increased specific mass of the solid, the interdendritic fluid flows mainly in the casting direction. It is nearly parallel to the surface of the slab and almost constant along a streamline. It is also at a maximum in the region where $g_{s}$ is at a minimum, i.e., near the centerline. Therefore, the velocity field obtained with shrinkage and bulging (Figure 5(b)) is somehow a superposition of the fields calculated in Figures 5(a) and 5(c): $\left(v_{l, x}-v_{s, x}\right)$ becomes then maximum and positive at the roll positions and is almost zero near the center of the section.

The contribution of shrinkage to the liquid velocity (Figure 5(c)) can be estimated from a simple mass balance. Assuming that $v_{l, y}=0$ everywhere in the mushy zone, the mass-conservation Eq. [8] can be simply integrated along the horizontal streamlines. It is easy to show that one has, at every point of the mushy zone,

$$
\rho_{s} g_{s} v_{c}+\rho_{l} g_{l} v_{l, x}=\text { cste }=\rho_{s} v_{c} \text { or } v_{l, x}-v_{c}=\left(\frac{\rho_{s}}{\rho_{l}}-1\right) v_{c}
$$

Using the values of Table I, the relative velocity of the fluid associated with shrinkage would be equal to $0.95 \mathrm{~mm}$ $\mathrm{s}^{-1}$, regardless of the value of $g_{s}$ (i.e., it is uniform). Such a relationship, which has already been deduced by Niyama for the prediction of porosity formation, ${ }^{[19]}$ only applies when the isovalues of the volume fraction of solid are perpendicular to the casting direction (i.e., Bridgman conditions). In the present case, $\left(\operatorname{grad} g_{s}\right)$ is nearly perpendicular to $\mathbf{v}_{c}$, and $\left(v_{l, x}-v_{c}\right)$ is clearly a function of $y$ : it is equal to $1.6 \mathrm{~mm}$ $\mathrm{s}^{-1}$ near the centerline and to 0 near the solid crust (Figure 5(c)). Please note that (1) the average of this shrinkageinduced velocity profile along a transverse section of the slab is about equal to the value given by Eq. [28] (0.95 mm $\left.\mathrm{s}^{-1}\right)$; (2) a small positive component of $\mathbf{v}_{l}$ along the $y$-axis compensates for shrinkage of deeper regions of the mushy zone (not clearly visible in Figure 5(c)). This small component partially offsets the negative value of $\mathbf{v}_{l}$ induced by bulging (Figure 5(a)). (Figure 5(d), showing the effect of soft reduction, will be explained later.)

At the same time the fluid flow is computed, the segregation map is also obtained according to the algorithm described in the previous section. The calculated carbonconcentration map of Figure 6 corresponds to the fluid-flow conditions of Figure 5(b), i.e., the calculation includes the shrinkage contribution and a maximum bulging of $1 \mathrm{~mm}$ between each section separating the six rolls. In this figure (and the following ones), the average solute content, $\bar{w}=$ $\left(w_{s} \rho_{s} g_{s}+w_{l} \rho_{l} g_{l}\right) / \bar{\rho}{ }^{*}$ is plotted. As the number of bulging

*It is easy to show that the mass fractions of solid and liquid ( $f_{s}$ and $f_{l}$, respectively) are equal to $\rho_{s} g_{s} \bar{\rho}^{-1}$ and $\rho_{l} g_{l} \bar{\rho}^{-1}$, respectively. Therefore, the average solute content $(\bar{w})$ is also equal to $w_{s} f_{s}+w_{l} f_{l}$.

events increases, solute-rich liquid is constantly pumped at the centerline by the successive expansion/compression
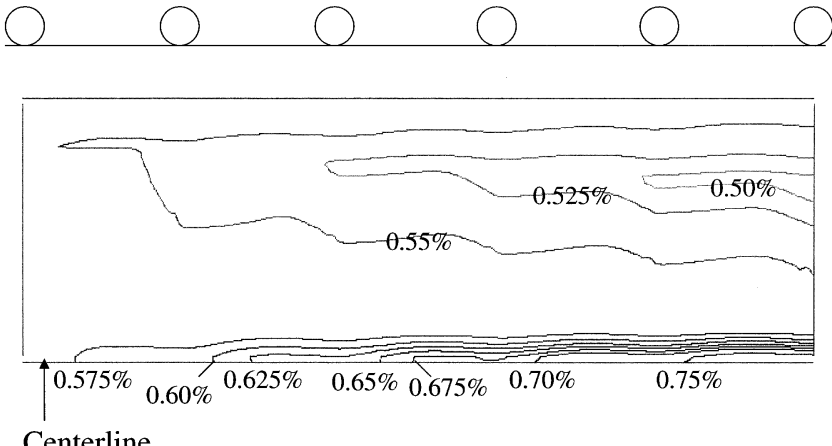

Casting direction

Fig. 6-Concentration map calculated for six rolls under the conditions of Fig. 5(b): $\rho_{s} \neq \rho_{l}$, maximum bulging of $1 \mathrm{~mm}$ between each pair of rolls; $g_{s}$ varies between 0.37 and 0.5 at the centerline.

sequences, while it is being expulsed from regions having higher volume fractions of solid. This results in a positive segregation near the centerline and a negative segregation closer to the slab surface. Please note that the concentration at the centerline increases from $0.55 \mathrm{wt}$ pct at the entrance of the domain to more than $0.8 \mathrm{wt}$ pct near the exit. Although shrinkage dramatically changes the relative fluid-flow velocity field (Figure 5), its effect on the overall segregation map is fairly small under stationary conditions. This can be understood by considering inverse segregation in directional solidification: ${ }^{[4,6]}$ the effect of shrinkage on macrosegregation is important near the surface of the chill and at the end of solidification, whereas during nearly steady-state conditions, the concentration is almost equal to the nominal concentration.

In order to see the small influence of shrinkage on segregation in continuous casting, two calculations were performed for a bulging displacement 10 times smaller $(0.1 \mathrm{~mm})$, with $\rho_{s}=\rho_{l}$ and $\rho_{s} \neq \rho_{l}$. The solute profiles obtained under such conditions are displayed along the centerline of the slab $(y=0)$ in Figure 7(a) and at the exit of the calculation domain $\left(x=L_{x}\right)$ in Figure 7(b). Without shrinkage (dashed curve), the "pumping" of solute near the centerline associated with each bulging event is clearly visible in Figure 7(a). It is decreased when shrinkage is considered (solid curve). The positive centerline segregation is accompanied by a depletion of solute in the peripheral regions of the slab, which were compressed during casting (Figure 7(b)). While solidification shrinkage also reduces the intensity of this negative segregation, it induces a second zone with a lower solute concentration near the centerline. The contribution of shrinkage alone (i.e., without bulging) is represented in Figure 7 as a dotted curve: it clearly induces a negative centerline segregation with a positive one in the periphery. If the relative fluid velocity induced by shrinkage (Figure 5(c)) was exactly parallel to $x$ everywhere in the mushy zone, its magnitude would be uniform and the contribution to macrosegregation would vanish under stationary conditions. However, as it is easier to feed the mushy zone from the centerline, where the volume fraction of solid is lowest, the velocity field in Figure 5(c) is not uniform, and there is a small positive $y$-component of the fluid flow associated with shrinkage, as noted before. This component is opposite that associated with bulging (Figure 5(a)) and induces a slight 


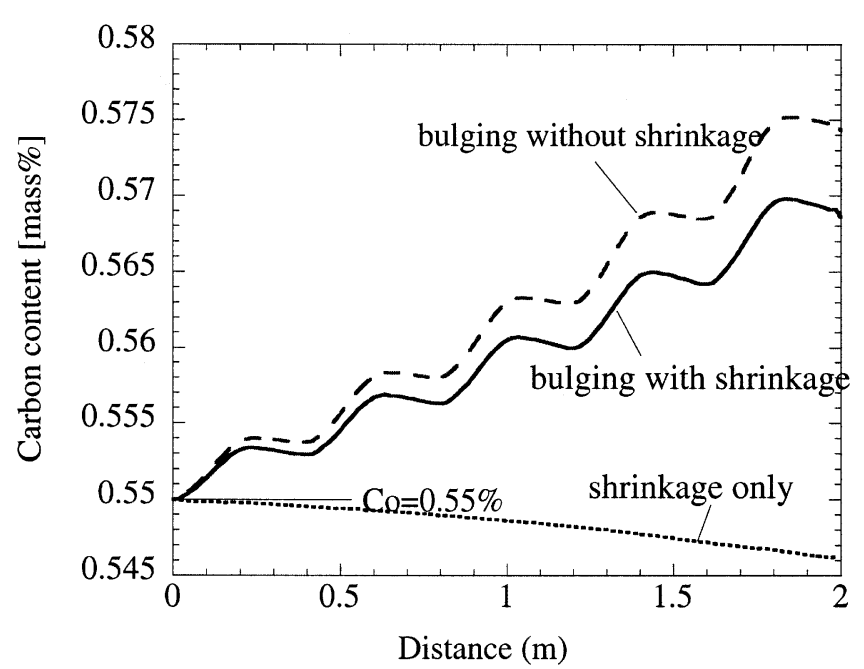

(a)

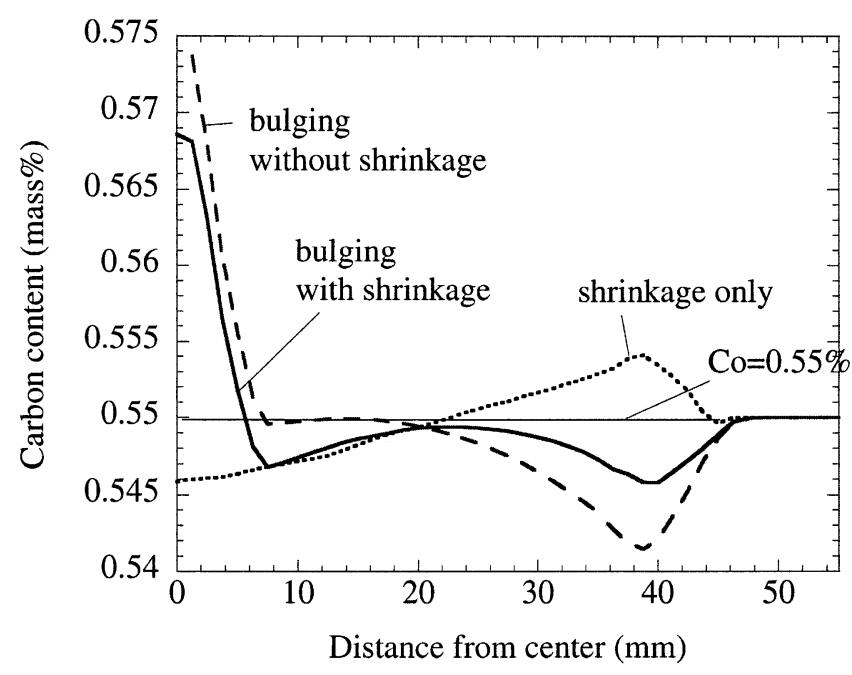

(b)

Fig. 7-Influence of the solidification shrinkage on the solute distribution (a) along the centerline and $(b)$ perpendicular to the centerline after six rolls $\left(g_{s, 0}=0.37\right.$, maximum bulging of $\left.0.1 \mathrm{~mm}\right)$. The segregation profile when only the shrinkage contribution is taken into account is also displayed (dotted line).

inverse segregation (i.e., negative centerline segregation). Please note that the solute content is conserved over the cross section of the slab for the three cases displayed in Figure 7(b): the integral of $\bar{w}$ over the cross section is equal to the nominal concentration $\left(w_{0}\right)$ times $L_{y}$. The negative centerline segregation reduced by shrinkage has been analyzed in details by Jalanti ${ }^{[20]}$ for the case of direct-chill continuous casting of aluminum alloys.

The effect of bulging on the centerline segregation is shown in Figure 8 as a function of the cast length. Clearly, the positive centerline segregation increases with an increasing displacement of the lateral surface of the slab. Please note that, in the absence of bulging $(0 \mathrm{~mm})$, there is a very small negative centerline segregation after six rolls, as already noted in the previous figure. This segregation is reversed with a bulging of only $0.1 \mathrm{~mm}$. Figure 9 shows the influence of the initial solid fraction $\left(g_{s, 0}\right)$ on the solute profile calculated after six rolls. The initial solid fraction is defined at

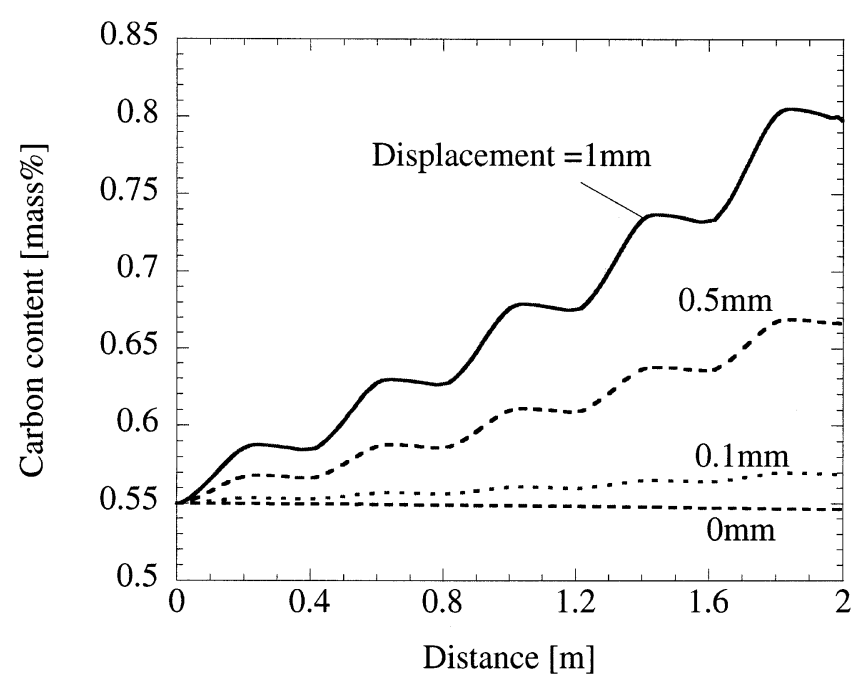

Fig. 8-Influence of the maximum bulging on the centerline segregation with shrinkage $\left(g_{s, 0}=0.37\right)$. Please note that in the absence of bulging, shrinkage induces a slight negative segregation at the center of the slab after six rolls.

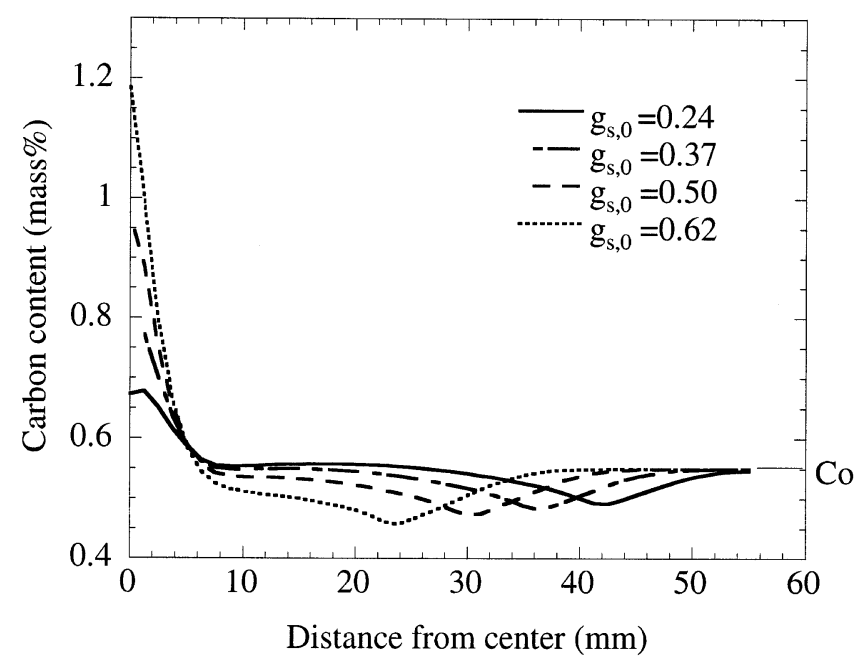

Fig. 9-Influence of the initial volume fraction of solid, $g_{s, 0}$, on the transverse solute profile calculated after six rolls (maximum bulging of $1 \mathrm{~mm}$, $\left.\rho_{s} \neq \rho_{l}\right)$.

the position $(0,0)$ of the calculation domain shown in Figure 3. As the solid fraction increases, the segregation between the peripheral and centerline zones is more pronounced. As the thickness of the mushy zone is reduced at the same time, the negatively and positively segregated zones become also closer. The enhancement of macrosegregation with an increase of $g_{s, 0}$ can be understood as follows: for a fixed bulging, the divergence of $\mathbf{v}_{s}$ increases with $g_{s, 0}$ as $\mathbf{v}_{s}$ varies over a smaller thickness of the mushy zone (in the compression area, Figure 4). At the same time, $g_{l}$ decreases. Both contributions in Eq. [8b] make the relative fluid-flow velocity to be larger, thus increasing the segregation intensity.

The calculated intensity of segregation for $g_{s, 0}=0.62$ is comparable to the value measured in Reference 21 . A central positive peak twice more than the nominal concentration is reported. Similarly, a negatively segregated region near the central peak has also been observed. ${ }^{[21]}$ It is clear that the 
present model is still semiquantitative, as the results depend on the number of rolls and could not be obtained for the entire length of the mushy zone. The CPU time is not the only reason, since the model could not be made to converge at a low or high volume fraction of solid. On the other hand, the bulging within each section of the casting machine should be calculated with detailed temperature-dependent mechanical properties in order to give quantitative results.

Next, the effect of soft reduction is investigated. Figure 5(d) shows the relative fluid-flow pattern calculated with an angle of $\alpha_{s r}=-1.510^{-4} \mathrm{rad}\left(-8.6 \times 10^{-3} \mathrm{deg}\right)$ in the presence of bulging and shrinkage. Such an angle is typically applied in the industrial process and allows one to precisely compensate for the shrinkage contribution. More specifically, this angle is such that the right-hand-side terms of the boundary conditions on $\partial \Omega_{2}$ and $\partial \Omega_{4}$ (Eqs. [20] and [21]) vanish, thus leaving conditions equivalent to those of a closed system (i.e., $\partial P / \partial n=0$ ). It is, therefore, not surprising that the liquid-flow pattern of Figure 5(d) is very similar to that calculated when considering bulging only (Figure 5(a)).

The solute distribution is, of course, modified also by soft reduction, as shown in Figure 10. With a maximum bulging of $0.1 \mathrm{~mm}$, it was already noticed that shrinkage reduced the positive centerline segregation (after six rolls and 0.1 $\mathrm{mm}$ of bulging, $\bar{w}\left(L_{x}, 0\right)$ decreases from 0.575 to $0.569 \mathrm{wt}$ pct when introducing shrinkage-Figure 7(a)). Surprisingly, when soft reduction is further applied to compensate for the shrinkage, the centerline segregation is even more decreased (after six rolls and 0.1 of mm bulging, $\bar{w}\left(L_{x}, 0\right)$ decreases from 0.569 to 0.564 wt pct when introducing soft reduction-Figure 10(a)). However, in the case of a maximum bulging of $1 \mathrm{~mm}$ (Figure 10(b)), the same soft-reduction angle that compensates for shrinkage exactly $-\alpha_{s r}$ - slightly increases segregation.

In fact, the simulation cases performed in the present contribution should be taken with some care for the following reason. In the case of no shrinkage and no soft reduction (Figure 5(a)), the conditions applied on all the boundaries of the domain $\Omega$, including $\partial \Omega_{2}$ and $\partial \Omega_{4}$, are those of a closed system (i.e., Eq. [13]), regardless of the bulging intensity. The same occurs when soft reduction is applied with an angle of $\alpha_{s r}$, which precisely compensates for shrinkage (Figure 5(d)). As a matter of fact, both situations result in a relative normal velocity of the fluid equal to zero at the vertical position of each roll. In these two situations, each section of the caster can be considered as an isolated domain (i.e., no relative exchange of mass between the sections), and, thus, "pumping" of solute from the peripheral zone to the centerline is simply adding at each section. In such cases, calculating one section or five sections of the continuous caster is representative: for given initial conditions of solute on $\partial \Omega_{4}$, the effect on the exit $\partial \Omega_{2}$ is found. When comparing the case of no shrinkage and no soft reduction (Figure 5(a)) and that of shrinkage compensated for by soft reduction (Figure 5(d)), it was found that soft reduction reduced shrinkage regardless of the maximum bulging.

On the contrary, when shrinkage is considered with a soft reduction that does not compensate exactly for shrinkage (including no soft reduction), there is a relative mass exchange between the various sections of the caster, as can be seen in Figures 5(b) and (c) (i.e., the relative velocity of

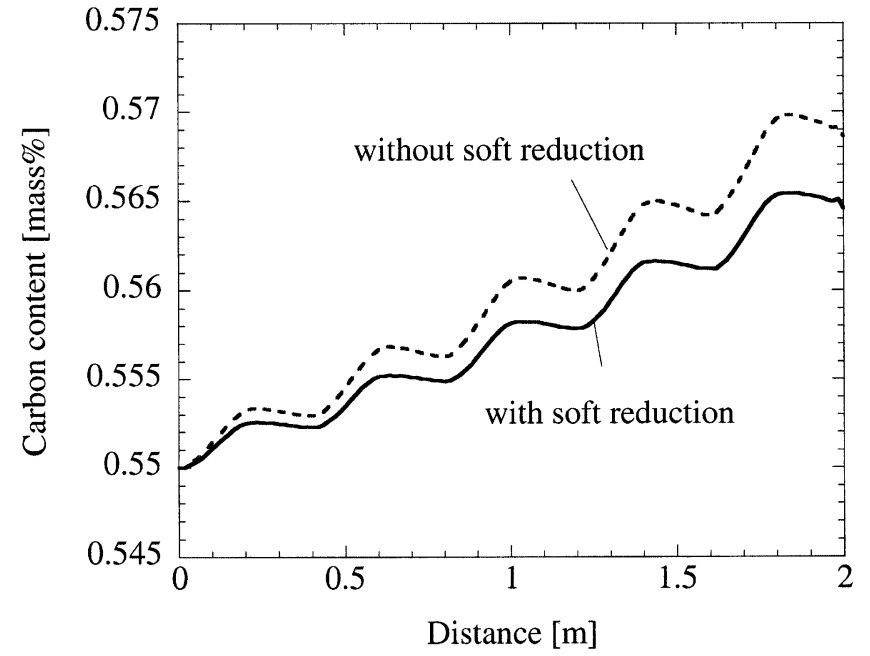

(a)

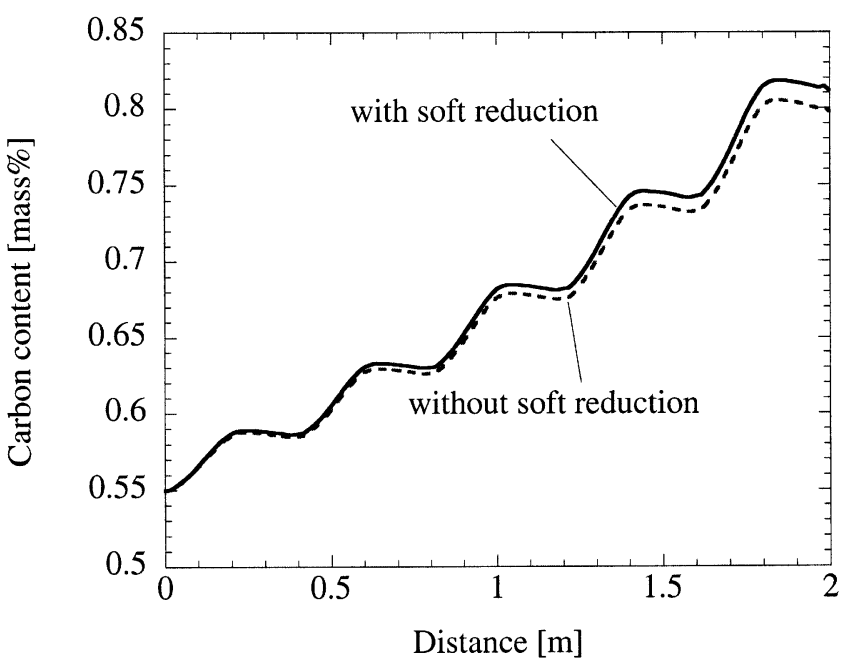

(b)

Fig. 10-Influence of soft reduction on the centerline segregation calculated with a maximum bulging of (a) $0.1 \mathrm{~mm}$ and, $(b) 1 \mathrm{~mm}\left(\alpha_{s r}=-1.5 \times\right.$ $\left.10^{-4} \mathrm{rad}, \rho_{s} \neq \rho_{l}\right)$.

the fluid at the vertical position of the rolls is not zero). Even though the slight negative segregation calculated at the centerline after six rolls in the absence of bulging and soft reduction (Figure 5(c)) is real, ${ }^{[20]}$ the values set on $\partial \Omega_{4}$ for the concentration field are not independent of the relative velocity of the fluid. In other words, when there is mass exchange between the sections of the caster, the calculation should be performed for the entire length of the mushy zone. This was not possible for two reasons: the computation time and the convergence problem encountered at low and high volume fractions of solid. As an indication, the computation time associated with six rolls was typically 2 hours on a SGI Origin $2000 \mathrm{R} 10000$ operating at $250 \mathrm{MHz}$, for an enmeshment including $121 \times 45$ nodal points. It took about 30 iterations to obtain convergence for all the equations (fluid flow, mass, and solute conservation equations). With such computation times, it is not unrealistic to envisage a calculation of deformation-induced macrosegregation performed over the 30- to 40-meter-long caster (i.e., including 
100 sections), providing the convergence at low and high values of $g_{s}$ is improved.

\section{CONCLUSIONS}

The deformation-induced macrosegregation during continuous casting of steel has been simulated using a fully Eulerian representation. Unlike the previous work of Miyazawa and Schwerdtfeger, ${ }^{[8]}$ the present model is based on a pressure formulation that respects the boundary condition at the centerline. Using a Lagrangian-based calculation of bulging performed with ABAQUS, this model employs a two-phase formulation of the mass and solute conservation equations. A Eulerian approach has the advantage over a Lagrangian representation, ${ }^{[9]}$ in that it is much easier to implement for several sections of a casting machine.

The present approach is fairly complete and has allowed the assessment, at least semiquantitatively, of the various contributions to centerline segregation by bulging, shrinkage, and soft reduction. It has still some limitations, which should be removed in order to provide a quantitative description. These points are as follows.

1. Simplistic but reasonable assumptions have been made for the deformation of the solid skeleton of the mushy zone, but a more refined approach could be used, e.g., the interpolation of the solid velocity in the compression areas could be different from a linear one. Some compression experiments of mushy zones deformed under wellcontrolled conditions should be performed before introducing more refined models.

2. As already stated, the model could not converge at volume fractions of solid lower than 0.2 or higher than 0.7 . As shown in Figure 9, centerline segregation at a low volume fraction of solid is fairly small. At a higher volume fraction of solid, bulging is certainly reduced as a result of an increased thickness and mechanical resistance of the fully solid crust, an improved coherency of the dendrites in the mushy zone, and maybe even bridging (coalescence) of dendrites located at the centerline. The problem then is strongly associated with the formation of hot tearing in such regions. This makes an estimation of bulging and other phenomena (e.g., metallostatic pressure and feeding) very difficult.

3. Thermal strains should also be included in the segregation model, as Lesoult and co-workers have shown that this effect could be fairly important. ${ }^{[9]}$ However, these authors did not consider specifically the rolls, which should decrease the importance of the thermal-strain contribution. A calculation of the bulging and thermal strains could be performed with ABAQUS prior to starting the present model, but a two-phase model is needed to calculate the influence on fluid flow and segregation. ${ }^{[22]}$

In the present study, solidification shrinkage alone was shown to induce a slight negative segregation at the centerline, as already predicted by Flemings and Nereo. ${ }^{[4]}$ This is due to the fact that $\operatorname{grad} g_{s}$ is not parallel to $\mathbf{v}_{s}$ : the interdendritic fluid preferentially flows, in this case, through the centerline region, where the permeability is larger. This induces a slight component of the fluid velocity away from the centerline and an associated negative centerline segregation. However, this contribution to segregation is fairly small as compared with the positive segregation induced by a normal bulging of $1 \mathrm{~mm}$.

When soft reduction exactly compensates for shrinkage, it has been shown that (1) each section of the caster behaves independently as a "pumping unit" of solute: solute-rich liquid is expulsed from the compression zone and results in positive centerline segregation under stationary conditions; and (2) the velocity field is quite close to the situation of no solidification shrinkage and no soft reduction (compare Figures 5(a) and (d)). This is true for the velocities located near the centerline; however, small differences occur near the roots of the dendrites. This is due to the slightly different thickness of the mushy zone over which the vertical component of the solid velocities is interpolated (Figure 4). As a result, shrinkage and soft reduction slightly reduces centerline segregation as compared with no soft reduction and no shrinkage (compare the dashed curve in Figure 7(a) with the continuous curve in Figure 10(a)).

When soft reduction does not compensate exactly for shrinkage (e.g., cases 5(b) and (c)), there is a relative mass exchange between the various sections of the caster, and the boundary conditions of solute at the entry of the domain are not easy to formulate, unless the whole caster length is considered. The result shown in Figure 10 should, therefore, be taken with some caution, as it is widely accepted in industry that soft reduction reduces centerline segregation. This effect was reproduced in the present simulations only for small amounts of bulging (Figure 10(a)), whereas a slightly opposite trend was found for larger amounts of bulging (Figure 10(b)).

\section{ACKNOWLEDGMENTS}

The authors acknowledge the financial support of Nippon Steel Corporation.

\section{REFERENCES}

1. W.D. Bennon and F.P. Incropera: Int. J. Heat Mass Transfer, 1987, vol. 30, pp. 2161-70, and 2171-87.

2. M. Ahmad, H. Combeau, J.-L. Desbiolles, T. Jalanti, G. Lesoult, J. Rappaz, M. Rappaz, and C. Stomp: Metall. Mater. Trans. A, 1998, vol. 29A, pp. 617-30.

3. M.R. Aboutalebi, M. Hasan, and R.I.L. Guthrie: Metall. Mater. Trans. $B, 1995$, vol. 26B, pp. 731-44.

4. M.C. Flemings and G.E. Nereo: Trans. TMS-AIME, 1967, vol. 239, pp. 1449-61.

5. S. Minakawa, I.V. Samarasekera, and F. Weinberg: Metall. Trans. B, 1985, vol. 16B, pp. 595-604.

6. P. Rousset, M. Rappaz, and B. Hannart: Metall. Mater. Trans. A, 1995, vol. 26A, pp. 2349-58.

7. C.Y. Wang and C. Beckermann: Metall. Mater. Trans. A, 1996, vol. 27A, pp. 2754-64.

8. K. Miyazawa and K. Schwerdtfeger: Arch. Eisenhüttenwes., 1981, vol. 52, pp. 415-22.

9. G. Lesoult and S. Sella: Solid State Phenomena, 1988, vols. 3-4, pp. 167-78.

10. K. Narita, T. Nozaki, T. Mori, K. Ayata, M. Onishi, and Y. Suzuki: Tetsu-to-Hagané, 1980, vol. 66, p. S791.

11. S. Ogibayashi, T. Mukai, M. Hirai, T. Tezuka, M. Yamada, and S. Mizoguchi: 7th Japan-Germany Seminar, Düsseldorf, May 1987, p. 309.

12. M.B. Li: Ph.D. Thesis, Ecole Centrale des Arts et Manufacture de Paris, Paris, 1989.

13. ABAQUS User Manual, Hibbitt, Karlsson \& Sorensen, Inc., Providence, RI, 1996.

14. J.B. Dalin and J.L. Chenot: Int. J. Num. Methods Eng., 1988, vol. 25, pp. 147-63. 
15. A. Grill and K. Schwerdtfeger: Ironmaking and Steelmaking, 1979, pp. 131-35.

16. P.F. Kozlowski, B.G. Thomas, J.A. Azzi, and H. Wang: Metall. Trans. A, 1992, vol. 23A, pp. 903-18.

17. Tekko-Binran, Seiko, Iron and Steel Institute of Japan, Tokyo, Japan, 1981, pp. 208-24.

18. S. Ganesan and D.R. Poirier: Metall. Trans., 1990, vol. 21B, pp. $173-81$.
19. E. Niyama, T. Uchida, M. Morioka, and S. Saito: AFS Int. Cast Met. J., 1982, Sept., pp. 52-63.

20. T. Jalanti: Ph.D. Thesis, Ecole Polytechnique Fédérale de Lausanne, Lausanne, Switzerland, 2000

21. Tekko-no-Gyoko, Iron and Steel Institute of Japan, Tokyo, Japan, 1977, p. 142.

22. I. Farup and A. Mo: Metall. Mater. Trans. A, 2000, vol. 31A, pp. 1461-72. 